\title{
A Simple Spreadsheet Program for the Calculation of Lattice-Site Distributions
}

\section{John G. McCaffrey}

Department of Chemistry, National University of Ireland-Maynooth, Maynooth, County Kildare, Ireland; john.mccaffrey@nuim.ie

Determination of lattice-site distributions-the number of lattice atoms or ions as a function of distance from a specific location-is essential in the calculation of numerous solid-state properties. Well-known examples include the Madelung constant, required for the determination of lattice energies (1) of ionic solids, or pair-distribution functions frequently encountered in statistical mechanics (2) and molecular dynamics (3) calculations. While the concept behind both parameters is relatively simple, most chemistry textbooks do not present the three-dimensional problem encountered in real systems. Those that do, especially in the calculation of lattice energies, truncate the series before the first mysterious "missing" lattice location is encountered and long before convergence (4) of the sum in the Madelung constant is achieved. Clearly, the actual determination of lattice-site distributions (5) is nontrivial even for practitioners. Attempting such a task within the constraints of undergraduate courses, where time is limited and where students often have little programming expertise, seems futile and as a result is infrequently undertaken.

A simple method to construct a spreadsheet program is presented that can be easily used in the calculation of latticesite distributions in physical chemistry laboratory or workshop sessions. The method used is based on the generation of everincreasing cubes and can be quickly set up by students with just an introductory knowledge of a spreadsheet program such as Excel. The method allows the prediction of accurate Madelung constants for cubic close-packed structures with the use of only a small (circa 10) number of cubes when the "zero-net-charge" condition is incorporated in the sum calculation. Algorithms (6, 7) created specifically for the evaluation of the Madelung constant unquestionably provide accuracy to many more decimal places, but the hands-on experience students gain in developing their own calculation more than compensates for this shortcom-

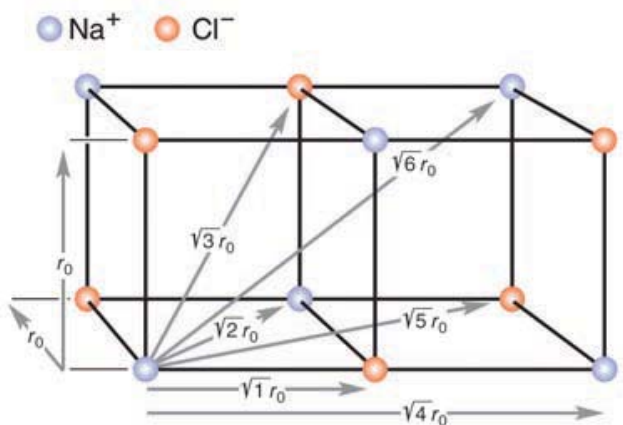

Figure 1. The shortest lattice distances in the $\mathrm{NaCl}$ structure are indicated as multiples of the nearest-neighbor distance, $r_{0}$. The values shown are easily determined by inspection using the Pythagorean theorem. The values presented in the diagram suggest the next distance term is $(\sqrt{ } 7) r_{0}$, a value that does not exist. The diagram also gives no idea of the number of ions located at these distances. ing. Moreover, the task of constructing the program requires students to actively consider and engage with the key governing factors allowing them to achieve a deeper understanding of the underlying principles.

Physical inorganic chemistry textbooks (1) develop the concept of a lattice-energy calculation along the lines of Figure 1, in which the lattice ion distances in $\mathrm{NaCl}$ are described using multiples of the nearest-neighbor distance, $r_{0}$. The energy, $E$, of the central sodium cation is then obtained by summing the terms whose denominators are the distances in Figure 1. This expression is frequently given for the potential energy of an ionic lattice

$$
\begin{aligned}
E=\frac{Z_{+} Z_{-} e^{2}}{4 \pi \varepsilon_{0} r_{0}} & \left(\frac{6}{\sqrt{1}}-\frac{12}{\sqrt{2}}+\frac{3}{\sqrt{3}}\right. \\
& \left.-\frac{6}{\sqrt{4}}+\frac{24}{\sqrt{5}}-\frac{24}{\sqrt{6}} \ldots\right)
\end{aligned}
$$

in which $Z_{+}$and $Z_{-}$are the ionic charges, $e$ is the charge on the electron, $\varepsilon_{0}$ is the permittivity of a vacuum, and the term in parentheses is the Madelung constant. From eq 1 the lattice points are located on spheres with relative radii represented by the denominators in the summation. With alternating signs and the denominator terms increasing in magnitude, a plausible argument can be presented that the infinite sum of eq 1 is a converging series. Several problems are inherent in this approach, however. The first is that after the second or third terms, students have no means of easily, but independently, establishing what the numerator values are, that is, the number of ions at specific multiples of the nearest-neighbor distance, $r_{0}$. The second is that the sum given by eq 1 is not convergent even when thousands of lattice ions are considered-a result that will be demonstrated later. The third and most problematic is that without prior knowledge, the student would reasonably expect, on the basis of what is shown in Figure 1, that the next term in the series is $(\sqrt{ } 7) r_{0}$. Actually, there is no ion in the $\mathrm{NaCl}$ structure at this distance! A major strength of the expanding-cube method for the determination of lattice-site distributions is the natural way in which the correct numbers of ions or atoms are present-or absent - at specific lattice distances.

Use of the spreadsheet program is illustrated in what may initially appear to be two unrelated systems. The first demonstrates its use in calculating the Madelung constant of $\mathrm{NaCl}$, while the second involves the prediction of the pair-distribution function for a neutral, van der Waals solid consisting of condensed krypton atoms. The pair-distribution function of the ionic solid will also be extracted revealing how this solid can be thought of as a pair of interlocking face-centered cubic cells. From an educational point of view, the method highlights concepts of conditional convergence and truncation errors inherent in numerical methods that must be considered by students when comparing their limited calculations with literature pair- 

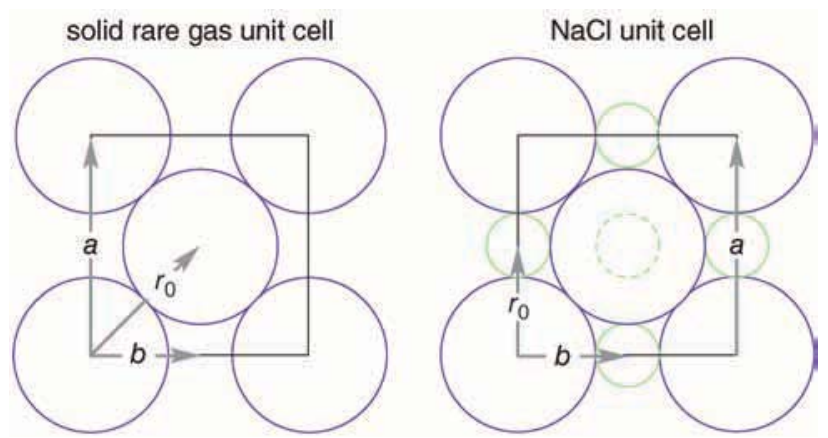

Figure 2. Space-filling models of the solid rare gases (left) and $\mathrm{NaCl}$ (right) lattices showing one face of the unit cells. As shown in the diagram, $b=a / 2$ in both cases where $a$ is the lattice parameter, the length of the edge of the unit cell. In contrast, the values of the nearestneighbor $\left(r_{0}\right)$ distances differ with $r_{0}=b$ for the $\mathrm{NaCl}$ lattice while for the solid rare gas lattice, $r_{0}=(\sqrt{ } 2) b$. The dotted circle shown on the right depicts the position of the $\mathrm{Na}^{+}$ion at the center of the $\mathrm{NaCl}$ unit cell. This ion is located one layer behind the face shown, at a distance $b=a / 2$. By coincidence, the lattice parameter of solid $\mathrm{Kr}$ ( $a=5.64 \AA)$ is the same as that of $\mathrm{NaCl}$, so the site size comparison made above is realistic.

distribution functions. In addition, the two systems chosen to illustrate the use of the method highlight both similarities and differences between the structure of an ionic lattice and a van der Waals solid consisting of neutral atoms. $\mathrm{NaCl}$ and solid krypton provide an interesting comparison as both have unit cells with virtually identical lattice parameters.

\section{Obtaining Lattice Distributions}

The distribution of lattice points with respect to a specific location in a cubic close-packed solid, such as the face-centered cubic, fcc, solid rare gases or an ionic solid whose lattice parameter is $a$, can be generated in ever increasing cubes and obtained by letting

$$
x=n_{1} b \quad y=n_{2} b \quad z=n_{3} b
$$

where $b=a / 2$ and the variables $n_{1}, n_{2}$, and $n_{3}=0, \pm 1, \pm 2$, $\pm 3, \ldots$. Figure 2 illustrates the relationship between the lattice parameter $a$, the edge length of the unit cell, and $b$, the distance term used in generating all the coordinates of the expanding cubes. For ionic solids, $b$ is the nearest-neighbor distance $\left(r_{0}=b\right)$ while for the solid rare gases $r_{0}=(\sqrt{2}) b$. Another important difference between the two types of lattices is that the sum of the coordinate indices $\left(n_{1}, n_{2}, n_{3}\right)$ can be even or odd for ionic lattices with

$$
\left|n_{1}\right|+\left|n_{2}\right|+\left|n_{3}\right|=0,1,2,3, \ldots
$$

while for the solid rare gases, the condition exists (5) that this sum can only be even,

$$
\left|n_{1}\right|+\left|n_{2}\right|+\left|n_{3}\right|=0,2,4,6, \ldots
$$

The distance, $r$, from the origin is determined as

$$
r^{2}=\left(n_{1}^{2}+n_{2}^{2}+n_{3}^{2}\right) b^{2}=\left(r^{\prime}\right)^{2} b^{2}
$$

where $r^{\prime}\left[=\left(n_{1}^{2}+n_{2}^{2}+n_{3}^{2}\right)^{1 / 2}=r / r_{0}\right]$ is a relative distance from the origin expressed as the multiple of the nearest-neighbor distance for an ionic solid. The ordinal number of each sphere of lattice points is $\left(n_{1}^{2}+n_{2}^{2}+n_{3}^{2}\right)$, that is, $\left(r^{\prime}\right)^{2}$ for an ionic solid.
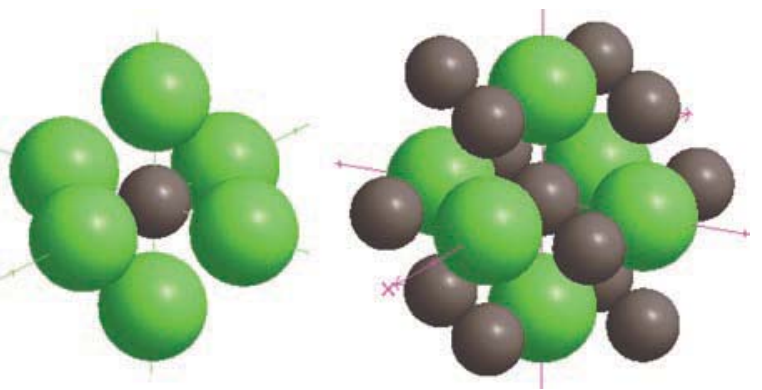

Figure 3. Positions of the chlorine anions making up the first sphere surrounding the central sodium cation in the $\mathrm{NaCl}$ structure are shown (left). The 6 chlorine anions are located as pairs on the Cartesian $x, y$, and $z$ axes. This corresponds to the centers of the 6 faces of the first surrounding cube. The structure at right is extended to include the 12 sodium cations in the second sphere. These atoms are located on the 12 edges of the first cube at $(\sqrt{ } 2) r_{0}$ from the central sodium cation.
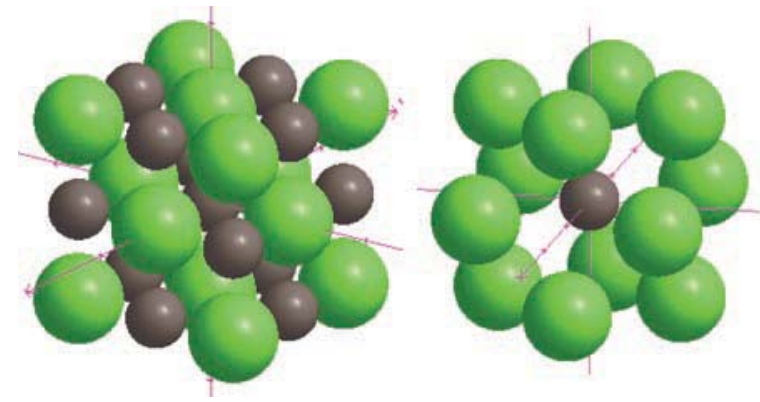

Figure 4. Positions of all ions in the first cube surrounding the central sodium cation in the $\mathrm{NaCl}$ structure are shown (left). The cube is completed with the addition of the $\mathrm{Cl}^{-}$anions located on the 8 corners of the first cube. Numbers of the ions present are 6,12 , and 8 at relative distances of $\sqrt{ } 1, \sqrt{ } 2$, and $\sqrt{ } 3$ from the central sodium cation at the origin. The corresponding fcc structure for a $\mathrm{Kr}$ lattice is shown (right) containing a neutral guest sodium atom at a substitutional site in this van der Waals solid for illustrative purposes. The structure is generated in the same way as the $\mathrm{NaCl}$ lattice but with the condition that sum of the indices can only be even-thus $\left|n_{1}\right|+\left|n_{2}\right|+\left|n_{3}\right|=$ $0,2,4,6, \ldots$ Comparing the two structures it becomes clear that the $\mathrm{Kr}$ atoms occupy only the second sphere positions of the $\mathrm{NaCl}$ structure, leaving vacancies at the $\mathrm{Cl}$ positions. In solid rare gases these vacancies are termed octahedral interstitial sites. Accordingly, only 12 nearest-neighbor atoms are contained within the first cube of the $\mathrm{Kr}$ lattice. The name given to the structure of the $\mathrm{NaKr}_{12}$ species is a cubo-octahedron.

The number of atoms at a given value of $r$ can be counted. This counting procedure can be conducted, with the assistance of Figure 3, for the first few surrounding spheres. Clearly all atoms in a given sphere are equidistant from the origin and it is the number of such atoms that determines the pair-distribution functions. In contrast, the first complete cube, consisting of three spheres, corresponds to the lattice unit cell.

As shown on the left in Figure 3 there are $6 \mathrm{Cl}^{-}$anions, with coordinates $( \pm 1,0,0),(0, \pm 1,0)$, and $(0,0, \pm 1)$, located at $1 r_{0}$ from the $\mathrm{Na}^{+}$ion at the origin. The next nearest-neighbor ions are sodium, which have the coordinates $( \pm 1, \pm 1,0),(0, \pm 1, \pm 1)$, and $( \pm 1,0, \pm 1)$, and are located at $(\sqrt{2}) r_{0}$ from the $\mathrm{Na}^{+}$ion. It is much less obvious than in the first sphere, but Figure 3 reveals there are $12 \mathrm{Na}^{+}$ions at $(\sqrt{ } 2) r_{0}$ from the central $\mathrm{Na}$ ion. Shown on the left in Figure 4 are the locations of the $\mathrm{Cl}^{-}$anions in sphere 3. As is 
evident in the diagram, the atoms in sphere 3 are located on the corners of the first complete cube, with coordinates $( \pm 1, \pm 1, \pm 1)$. Being on the corners of a cube, they must be 8 in number. This counting method is convenient for only the ions in three spheres contained within the first cube, generated from the $\left(n_{1}, n_{2}, n_{3}\right)$ index values $(1,0,0),(1,1,0)$, and $(1,1,1)$. Extending this counting procedure beyond the first cube is a tedious activity and a procedure that is extremely error prone. Consequently, an alternative approach must be employed to allow students to conduct reliable calculations with ease for expanded cubes.

Fortuitously the pair-distribution function depends only on the numbers of atoms or ions at specific distances and has no dependence on their position (above-below, left-right, etc.) with respect to the central point. As a result, extended calculations of lattice-site distributions introduce the idea of multiplicity for all the $\left(n_{1}, n_{2}, n_{3}\right)$ index values encountered in the cubes generated. Usage of the multiplicity term, multip, given by the formula

$$
\text { multip }=\frac{3 !}{\left(4-N_{\text {diff }}\right) !} 2^{N_{\text {nonzero }}}
$$

greatly facilitates the calculation of expanded cubes. It has already been utilized in a recent article ( 8 ) in this Journal, so only the identification of its two component terms will be given here. Briefly, $N_{\text {diff }}$ is the number of different absolute values in a selected $\left(n_{1}, n_{2}, n_{3}\right)$ index. In the first combination $(1,0,0)$ for instance, this term will be 2 , because there are two different absolute values of $n_{1}, n_{2}, n_{3}$, namely, 1 and 0 . As indicated in Appendix A (see the online material), this mathematical condition can easily be programmed in Excel with the logical IF statement $\operatorname{IF}(\mathrm{AND}(\mathrm{A} 3=\mathrm{B} 3, \mathrm{~B} 3=\mathrm{C} 3), 1, \operatorname{IF}(\mathrm{OR}(\mathrm{A} 3=\mathrm{B} 3, \mathrm{~B} 3$ $=\mathrm{C} 3), 2,3)$ ) when the three absolute values of $n_{1}, n_{2}$, and $n_{3}$ are entered in the cells A3, B3, and C3. $N_{\text {nonzero }}$ is simply the number of nonzero values in a given index. For instance, the index $(1,0,0)$ has a value of 1 for $N_{\text {nonzero }}$. This condition is programmed as $\operatorname{IF}(\mathrm{AND}(\mathrm{B} 3=0, \mathrm{C} 3=0), 1, \operatorname{IF}(\mathrm{AND}(\mathrm{B} 3>0, \mathrm{C} 3=0), 2,3))$. Using the results returned for $N_{\text {diff }}$ and $N_{\text {nonzero }}$ in cells G3 and H3, respectively, the multip term is evaluated according to eq 6 with $\left.\left(\mathrm{FACT}(3)^{*} 2^{\wedge} \mathrm{H} 3\right) / \mathrm{FACT}(4-\mathrm{G} 3)\right)$. Calculation shows that for a numbering system having three indices, multip can only have five possible values, namely $6,12,8,24$, and 48 .
With these programming statements in place the procedure of developing calculations for expanded cubes can begin. Appendix A provides a complete listing of the programming statements even though students can come up with their own expressions. The three possible indices encountered in generating the first cube have already been given: $(1,0,0),(1,1,0)$, and $(1,1,1)$. Based on this pattern the students can generate the second cube indices as $(2,0,0),(2,1,0),(2,1,1),(2,2,0),(2,2,1)$, and $(2,2,2)$. An indication of how the spreadsheet may appear when this has been done is given in Table 1 . When all the indices of the second cube have been correctly entered, students can then embark upon independently entering all the combinations of $n_{1}, n_{2}$, and $n_{3}$ up to a value of 10 . Experience shows this can be achieved in the first quarter of a typical two-hour computer laboratory or workshop session.

\section{Predicting Madelung Constants}

The importance of the Madelung constant has been highlighted in several articles $(9,10)$ in this Journal with the most recent discussion that presented by Vining and co-workers (8). These authors have also presented a program (11), available through JCE Software, for the calculation of lattice energetics. Description and discussion of the importance of conditional convergence in calculating the Madelung constant is given in Vining's recent JCE article (8), Winn's Physical Chemistry textbook (12), or Solid State Physics by Kittel (13). For space reasons, only the details of the spreadsheet calculations will be presented here. The method ultimately implemented in the present calculation is based on the zero-net-charge (or neutrality ${ }^{1}$ ) condition for expanding cubes (14).

Before implementing the zero-net-charge condition it is, however, instructive to attempt a "crude" sum of the Madelung constant according to eq 1 . This is done by simply adding the terms in the rightmost column of Table 1 and multiplying by -1 (see Appendix A in the online material). The constants evaluated by performing this sum are represented in Figure 5 as squares connected by a dashed line. It is evident in Figure 5 that convergence is not occurring up to the 10th cube, even though a total of 4630 atoms have already been considered at this stage. This is the method represented by eq 1 and an approach that clearly is not providing the expected convergence.

Table 1. An Excel Spreadsheet Workpage Used To Evaluate the Distances and the Numbers of the lons (Multip) Contained within the First 2 Cubes of the NaCl Lattice

\begin{tabular}{|cccccccccc|}
\hline$\left|n_{1}\right|^{a}$ & $\left|n_{2}\right|$ & $\left|n_{3}\right|$ & $\Sigma\left|n_{i}\right|$ & $\left(\left.r^{\prime}\right|^{2 b}\right.$ & $\operatorname{Sign}^{c}$ & $N_{\text {diff }}$ & $N_{\text {nonzero }}$ & Multip & Sign $\times$ Multip/ $r^{\prime d}$ \\
\hline 1 & 0 & 0 & 1 & 1 & -1 & 2 & 1 & 6 & -6 \\
1 & 1 & 0 & 2 & 2 & 1 & 2 & 2 & 12 & 8.485281 \\
1 & 1 & 1 & 3 & 3 & -1 & 1 & 3 & 8 & -4.6188 \\
2 & 0 & 0 & 2 & 4 & 1 & 2 & 1 & 6 & 3 \\
2 & 1 & 0 & 3 & 5 & -1 & 3 & 2 & 24 & -10.7331 \\
2 & 1 & 1 & 4 & 6 & 1 & 2 & 3 & 24 & 9.797959 \\
2 & 2 & 0 & 4 & 8 & 1 & 2 & 2 & 12 & 4.242641 \\
2 & 2 & 1 & 5 & 9 & -1 & 2 & 3 & 24 & -8 \\
2 & 2 & 2 & 6 & 12 & 1 & 1 & 3 & 8 & 2.309401 \\
\hline
\end{tabular}

${ }^{a}\left|n_{1}\right| \geq\left|n_{2}\right| \geq\left|n_{3}\right|$. 'DTe fifth column correctly reveals the absence of an ion $r^{\prime}=\sqrt{7}$. The absences at $r^{\prime}=\sqrt{ } 10$ and $\sqrt{ } 11$ are artifacts arising from a calculation limited to the second cube. This error is corrected when the calculation is expanded to include the third cube. "Sign is given by $(-1)^{\left(\left|n_{1}\right|+\left|n_{2}\right|+\left|n_{3}\right|\right)}$; for $\mathrm{NaCl}$ it represents the charge of ions in a given sphere. ${ }^{\mathrm{d}}$ To obtain positive Madelung values, all the multip/ $r^{\prime}$ terms should be multiplied by -1 as indicated in Appendix A (see the online material). 
This lack of convergence behavior is well known and can be corrected by summing the interactions for cubes with zero net charge. This condition is achieved by allowing the ions at the corner, edge, and face positions of the cubes to contribute fractional charges of $1 / 8,1 / 4$, and $1 / 2$, respectively. These specific values can be programmed in Excel with the logical "IF" statement as follows: $\operatorname{IF}(\mathrm{AND}(\mathrm{A} 3=\mathrm{B} 3, \mathrm{~B} 3=\mathrm{C} 3), 0.125, \operatorname{IF}(\mathrm{AND}$ $(\mathrm{A} 3=\mathrm{B} 3, \mathrm{~B} 3>\mathrm{C} 3), 0.25,0.5))$. As before the absolute values of the three index values $n_{1}, n_{2}$, and $n_{3}$ are contained in cells A3, $\mathrm{B} 3$, and $\mathrm{C} 3$. When the value returned by the above condition (although not shown in Table 1 for space reasons) is multiplied by the sign $\times$ multip $/ r^{\prime}$ term and then summed, the result of the zero-net-charge condition is available. Figure 5 provides comparison of this result, shown by the diamonds, with the crude sum, shown by the squares. The vast improvement obtained in the convergence behavior when the zero-net-charge condition is imposed is immediately evident.

Figure 6 provides a zoomed-in view of the conditionally converged series in which it emerges that after the 5 th cube, the Madelung value oscillates in the small range between 1.7475 and 1.7476. At the 10th cube, the Madelung value is evaluated as 1.747568 while the generally accepted value is 1.74756 (15). A more exact value of 1.747564594 has recently been provided $(6,16)$. During a workshop or laboratory session, little improvement of the Madelung constant will be gained by students spending additional time to extend their spreadsheet calculation beyond the 10th cube.

\section{Predicting Pair-Distribution Functions}

\section{lonic Lattice}

The calculations undertaken in determining the Madelung constant have already utilized the lattice-site distributions of the ions, but because only the sum of the terms is involved, the pairdistributions function, $n(r)$ - the numbers of ions at specific lattice distances-is not immediately at hand. To obtain $n(r)$, the distances obtained for all the indices considered up to the $i$ th cube must be sorted in order of increasing $r$ and the multiplicities of any identical $r$ values summed. For example, the lattice indices $(3,2,2)$ and $(4,1,0)$ both give rise to a distance of $(\sqrt{ } 17) r_{0}$, so their multiplicities (both equal to 24 ) must be summed to obtain the correct contribution to radial distribution at this distance. Rather than do this check manually, a few lines of computer code can be written to check for equal adjacent values in the presorted array of $r$, multip. When a pair is found, the two $y$ values are summed and one of the $r$ 's is discarded. This summing procedure must be done three times to remove all the repeat $r$ 's that occur up to the 10th cube. The code required to do this is given in Appendix B in the Genplot (17) programming language (see the online material). All subsequent data plots were generated with this plotting and data analysis software.

The result of such a pair-distributions calculation is shown in panel A of Figure 7 in terms of the number of ions in the first 8 spheres surrounding the central ion. The numbers of ions involved are 6, 12, 8, 6, 24, 24, 0, and 12. As shown in Table 1, the expanding-cube method correctly identifies the absence of an ion at $(\sqrt{ } 7) r_{0}$. The results shown there would also suggest the absence of ions at $(\sqrt{ } 10) r_{0}$ and $(\sqrt{ } 11) r_{0}$. However, when the calculation is extended into the third cube it immediately becomes evident that the latter absences arise from the limited number of sites considered in the first two cubes. This observation provides an important reminder that the pair-distribution function is a radial function. It can be considered as an early warning that serious truncation errors will occur without due consideration of the cube number under calculation. As shown in panel B of Figure 7 , the next true absence occurs at $3.873,(\sqrt{ } 15) r_{0}$, when the calculation is extended into subsequent cubes. The more extended calculation presented in panel B is shown as a function of relative radial distance, $r^{\prime}$, rather than the sphere number used in panel A. For the fcc lattice of $\mathrm{NaCl} r_{0}$ is, as shown in Figure 2 , half the lattice parameter, $a,\left(r_{0}=a / 2\right)$.

To generate meaningful pair-distributions functions, $n\left(r^{\prime}\right)$, from the spreadsheet calculation done up to and including the 10th cube of the $\mathrm{NaCl}$ lattice, the following consideration must be included. Even though the calculation extends to the 8 corners of this cube-with coordinates $(10,10,10)$ and thereby corresponds to a maximum distance of $17.32,(\sqrt{300}) r_{0}$, the $n\left(r^{\prime}\right)$ values obtained are only reliable out to the center of the face of the 10 th cube $(10,0,0)$. The 6 atoms in this complete sphere are located at a distance of $(\sqrt{ } 100) r_{0}$ from the origin. Because of the truncation errors that arise beyond this distance, the plot shown in panel $\mathrm{B}$ is limited to a distance of 10 nearest neighbors from

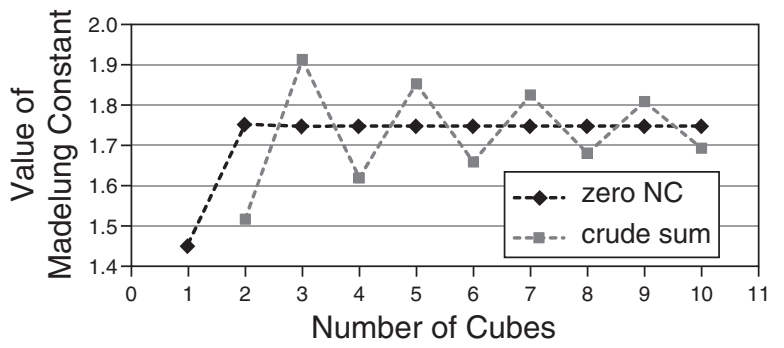

Figure 5. A comparison of the Madelung constants evaluated by two methods within the first 10 cubes surrounding the central sodium cation in the $\mathrm{NaCl}$ structure. The results of a crude sum of the terms in the last column of Table 1 are shown by the squares, while those obtained as a sum of cubes complying with zero-net-charge condition are shown by the diamonds. The comparison reveals the requirement of the zero-net-charge (charge neutrality) condition in obtaining a converged value of the Madelung constant.

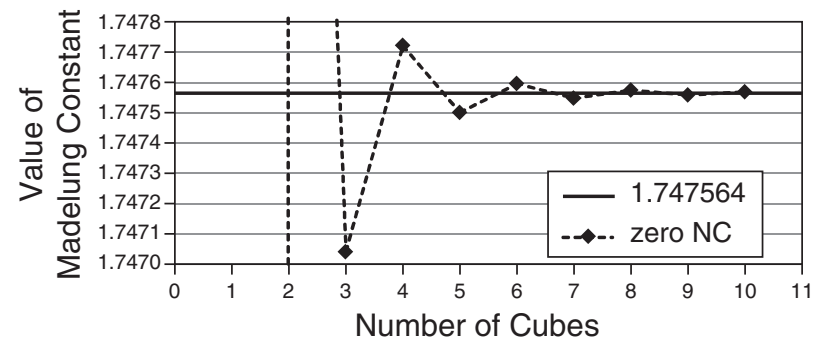

Figure 6. The Madelung constants evaluated in the spreadsheet calculation by imposing the zero-net-charge (or charge neutrality) condition. It is evident from the plot that the results obtained after the 5 th cube have converged to 4 decimal places. The Madelung constant of $\mathrm{NaCl}$, exact to six decimal places, 1.747564, is shown by the solid line revealing the high level of convergence that exists after the 7th cube. In the present expanding-cube method, the Madelung constant is evaluated as 1.747568 at the 10th cube with the zero-net-charge condition. 
the origin. As expected, $n\left(r^{\prime}\right)$ increases as the distance between the origin and the surrounding spheres becomes larger. It actually increases more rapidly than it may appear in the figure because of the distances between adjacent spheres becoming smaller and smaller. The more usual way (2) of presenting the pair-distribution function is to divide the $n\left(r^{\prime}\right)$ values by the volume element $4 \pi\left(r^{\prime}\right)^{2} \mathrm{~d} r^{\prime}$. Such a plot is shown in panel C of Figure 7 for the same distance range depicted in panel $\mathrm{B}$. This treatment has the effect of emphasizing the importance of the atoms and ions in the nearest surrounding spheres. In panel D the first 16 surrounding spheres of the $\mathrm{NaCl}$ lattice are depicted as "stick spectra" and the pair distribution is shown as a function of the actual distance in a range up to $12 \AA$ from the $\mathrm{Na}^{+}$ ion at the origin. Superimposed on this is the pair-distribution function that will exist in reality where small variations exist in the lattice positions owing to thermal motion of the ions. This thermal broadening will be present in a molecular dynamics (MD) calculation of the solid lattice (18). A positional variation of $1 \%$ of the lattice parameter is shown, corresponding to a temperature of approximately $1000 \mathrm{~K}$. In MD calculations the pair distribution is calculated numerically in a selected radial distance range, $\Delta r$. The result $G(r)$ is presented in a manner similar to that described above.

$$
G(r)=\frac{n(r)}{\sigma 4 \pi r^{2} \Delta r}
$$

The additional parameter, $\sigma$, is a symmetry factor that has a value of 2 for calculations of identical species, for example, the
$\mathrm{Na}^{+}-\mathrm{Na}^{+}$pairs (or in a homogeneous lattice) and a value of 1 for distinct pairs, for example, $\mathrm{Na}^{+}-\mathrm{Cl}^{-}$(or a guest atom in a host lattice). The gray curve in panel $\mathrm{D}$ shows the lattice-site distributions for the $\mathrm{Na}^{+}-\mathrm{Cl}^{-}$pairs while the black curve depicts the $\mathrm{Na}^{+}-\mathrm{Na}^{+}$pairs.

\section{Neutral (van der Waals) Lattice}

Determining the lattice-site distribution of a van der Waals solid is conducted with the same expanding-cube method as that outlined for the ionic lattice, with the one important difference, which is that sum of the indices can now only be even (5). This arises from the condition, mentioned earlier, that for the fcc lattice of a solid consisting of neutral species, the index sum $\left|n_{1}\right|+\left|n_{2}\right|+\left|n_{3}\right|=0,2,4,6, \ldots$, which has the effect that the odd terms present in the $\mathrm{NaCl}$ lattice are now absent. In the spreadsheet calculation the odd or even characters can be identified most conveniently with the sign of the term $(-1)^{\left(\left|n_{1}\right|+\left|n_{2}\right|+\left|n_{3}\right|\right)}$ used in the Madelung constant calculation. Equation 7 is incorporated in the spreadsheet program with the following modification $\operatorname{IF}\left(\mathrm{F} 3=1,\left(\mathrm{FACT}(3) * 2^{\wedge} \mathrm{H} 3\right) / \mathrm{FACT}(4-\mathrm{G} 3), 0\right)$, which utilizes the sign of the term in the cell F3. As indicated in Appendix C ( see the online material), this logical IF statement returns a multip value of 0 for all terms except even ones. Equation 6 is used to evaluate the multiplicities of the even terms in the usual way. The worksheet resulting for the first two cubes is presented in Table 2.

As is evident in the table, roughly every other one of the spheres present in the ionic lattice is absent in the neutral lattice. Connected with this site absence, for the neutral solid $r_{0}=a / \sqrt{ } 2$
Figure 7. The lattice-site distribution for $\mathrm{NaCl}$ calculated by the expandingcube method. In panel $A$ the number of ions in the first 8 surrounding spheres are shown, revealing the absence of a 7 th sphere. The relative distance $r^{\prime}$ is shown in panel $B$. This panel shows the distribution $n$ as a function of $r^{\prime}$. Panel $C$ shows the $n\left(r^{\prime}\right)$ function divided by the volume element $4 \pi\left(r^{\prime}\right)^{2} d r^{\prime}$, which emphasizes the importance of the spheres closest to the central ion. The stick spectrum in panel $D$ shows the same distribution function but now in terms of actual distance from the central sodium cation. This is obtained by multiplying $r^{\prime}$ (shown in panel C) by the value of the distance between nearest neighbors $b$ $(=a / 2)$. The curves in panel $D$ shows the pair-distribution function convoluted by a Gaussian function to simulate the positional variation of the lattice sites caused by thermal motion. This is how the results of a molecular dynamics evaluation of pair-distributions are presented. For the purpose of illustration the positions of the $\mathrm{Na}^{+}-\mathrm{Cl}^{-}$pairs are shown by the gray curves while the $\mathrm{Na}^{+}-\mathrm{Na}^{+}$pairs are shown in black.
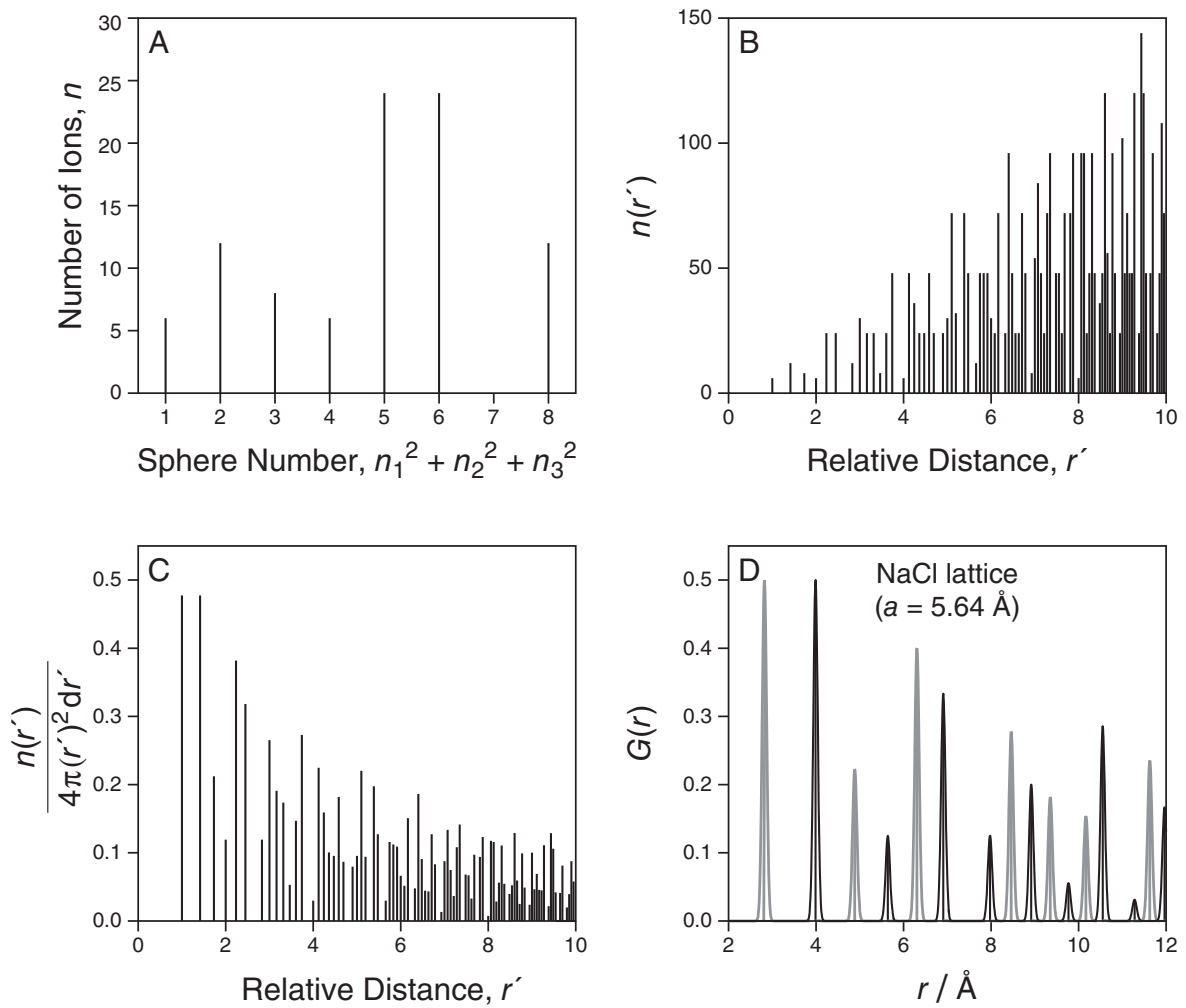
and not $a / 2$ as in the ionic lattice. Equation 5 then becomes

$$
r^{2}=\left(r^{\prime}\right)^{2} b^{2}=\left(r^{\prime}\right)^{2}\left(\frac{r_{0}}{\sqrt{2}}\right)^{2}
$$

and the relative distance is

$$
\frac{r}{r_{0}}=\frac{r^{\prime}}{\sqrt{2}}
$$

Panel A of Figure 8 presents the first eight nearest-neighbor surrounding spheres of a van der Waals lattice revealing the presence of $12,6,24,12,24,8,48$, and 6 atoms in spheres 1 through 8 . Consistent with the findings of previous investigations (5), the expanding-cube method locates the first absence in the van der Waals lattice at sphere number 14; the next one occurs at sphere number 30. When the pair-distribution function is plotted against relative distance $\left(=r^{\prime} / \sqrt{2}\right)$ as shown in panel $\mathrm{B}$, these absences are located at 3.74 and 5.477 , respectively, with the next absence evident at $6.78\left[\left(r^{\prime}\right)^{2} / 2=46\right]$.

At this point a note of caution is necessary because with the increased nearest-neighbor distance in the van der Waals lattice, truncation errors arise earlier in this system than the limit of 10 identified in the ionic lattice. The "cut-off" radius is now identified as a relative distance of 7.07, $[=(\sqrt{100 / 2})]$. Because of this, the pair-distribution results obtained from evaluating 10 cubes are limited to the relative distance of 7 shown in panels $\mathrm{B}$ and $\mathrm{C}$ of Figure 8 . The smooth black curve shown in panel D of Figure 8 presents the pair-distribution function convoluted by a Gaussian function to simulate the positional variation of the
Table 2. An Excel Spreadsheet Workpage Used To Evaluate the Distances and the Numbers (Multip)

of the Neutral Atoms Contained within the First 2 Cubes of a van der Waals Lattice Such as the Solid Rare Gases

\begin{tabular}{|ccccccccc|}
\hline$\left|n_{1}\right|$ & $\left|n_{2}\right|$ & $\left|n_{3}\right|$ & $\Sigma\left|n_{i}\right|$ & $\left(r^{\prime}\right)^{2}$ & Sign & $N_{\text {diff }}$ & $N_{\text {nonzero }}$ & Multip \\
\hline 1 & 0 & 0 & 1 & 1 & -1 & 2 & 1 & 0 \\
1 & 1 & 0 & 2 & 2 & 1 & 2 & 2 & 12 \\
1 & 1 & 1 & 3 & 3 & -1 & 1 & 3 & 0 \\
2 & 0 & 0 & 2 & 4 & 1 & 2 & 1 & 6 \\
2 & 1 & 0 & 3 & 5 & -1 & 3 & 2 & 0 \\
2 & 1 & 1 & 4 & 6 & 1 & 2 & 3 & 24 \\
2 & 2 & 0 & 4 & 8 & 1 & 2 & 2 & 12 \\
2 & 2 & 1 & 5 & 9 & -1 & 2 & 3 & 0 \\
2 & 2 & 2 & 6 & 12 & 1 & 1 & 3 & 8 \\
\hline
\end{tabular}

Note: The large number of lattice absences, indicated by multip $=0$, arise from the even-only condition that exists for the index sum ||$\left.n_{1}|+| n_{2}|+| n_{3} \mid=0,2,4, \ldots\right)$ of the fcc lattices formed by van der Waals solids.

lattice sites caused by thermal motion. The abscissa used in this plot is actual distance of the Kr lattice in Ångstrom units. Using the lattice parameter of solid $\mathrm{Kr}$ as $5.64 \AA$, panel D presents how the results of a molecular dynamics evaluation of $\mathrm{Kr}-\mathrm{Kr}$ pair distributions will appear. For the purpose of illustration, a $2 \%$ positional variation of the lattice parameter is shown, corresponding to a temperature of approximately $10 \mathrm{~K}$.

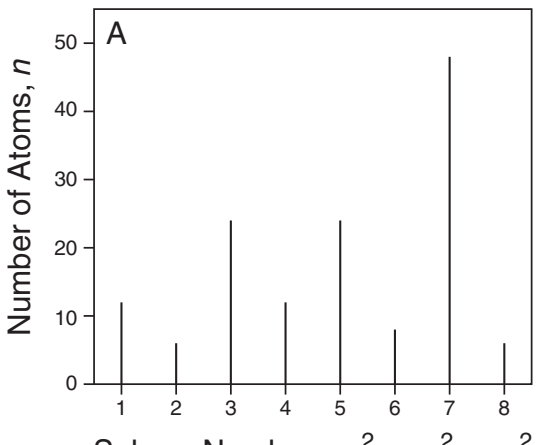

Sphere Number, $n_{1}^{2}+n_{2}^{2}+n_{3}^{2}$

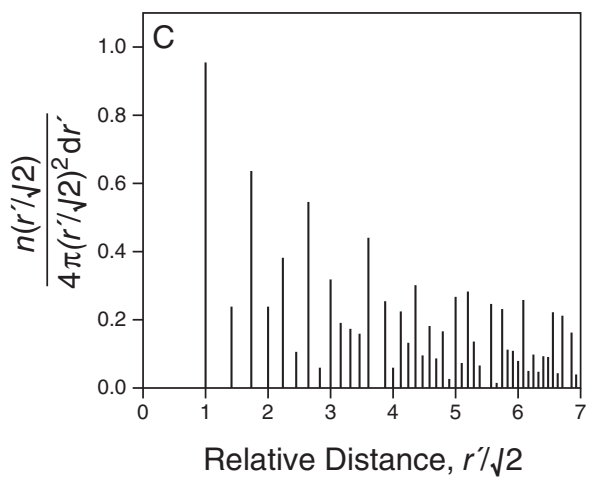

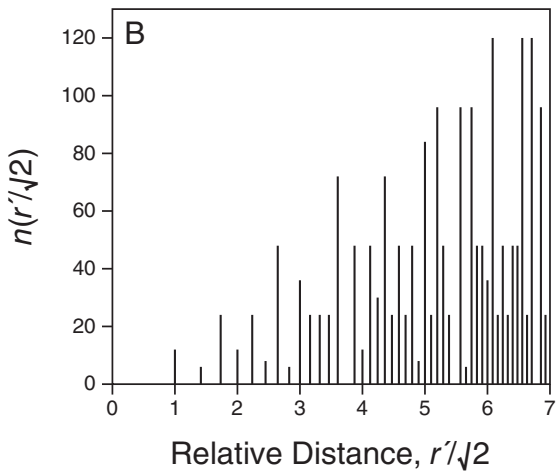

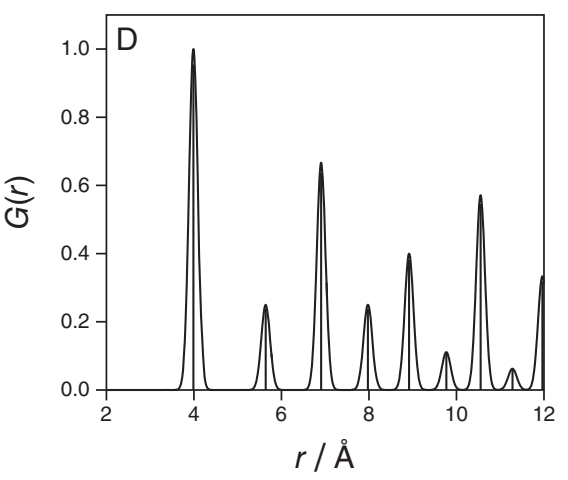

Figure 8. Calculation of the pairdistribution function of a van der Waals solid as determined by the expandingcube method. The treatment is the same as for $\mathrm{NaCl}$ but because of the imposition of the index sum as even, only the terms satisfying the condition ||$\left.n_{1}|+| n_{2}|+| n_{3} \mid=0,2,4, \ldots\right)$ exist. 
The fcc solids formed by $\mathrm{NaCl}$ and solid krypton both have the same lattice parameters $a=5.64 \AA$. Panels D in Figures 7 and 8 present the results of the present pair-distribution calculations for $\mathrm{NaCl}$ and solid $\mathrm{Kr}$, respectively, in the same distance range of 2-12 $\AA$. Comparing these two panels reveals how different the two lattices are. In Figure 8 it is clear just how much more open the $\mathrm{Kr}$ lattice is compared with that shown in Figures 7 for the $\mathrm{NaCl}$ lattice. The key difference between the two lattices is that the sites occupied by the smaller $\mathrm{Na}$ cations in the $\mathrm{NaCl}$ lattice are vacant in the $\mathrm{Kr}$ lattice. These intrinsic vacancies are referred to as octahedral interstitial $\left(I_{\mathrm{Oh}}\right)$ sites and in the solid rare gases are usually too small to accommodate guest atomic species in their ground states. However, calculations (19) show that the excited electronic states of guest metal atoms transiently migrate in these $I_{\mathrm{Oh}}$ sites prior to photon emission.

An alternative description of the packing in the $\mathrm{NaCl}$ lattice becomes evident in panel D of Figure 7 in which the structure can be viewed as a pair of interwoven fcc lattices. The compact nature of this lattice and the strong electrostatic bonding between the ion pairs ensures that elevated temperatures $(>1000 \mathrm{~K})$ must be reached before appreciable deviation will occur from the lattice-site distributions (18). In contrast, MD calculations (20) find that large deviations already exist in the solid $\mathrm{Kr}$ lattice at even $10 \mathrm{~K}$.

\section{Conclusions}

The expanding-cube method allows for straightforward programming in a spreadsheet that can be developed independently by students to calculate accurate Madelung constants and predict the pair-distribution functions of ionic or van der Waals solids. During the course of this work students are exposed to ideas such as the requirement of zero net charge to obtain rapid convergence of the sum done in the determination of the Madelung constant. In the evaluation of pair-distribution functions, issues arise concerning the identification of reliable cut-off radii to avoid truncation errors. Solid $\mathrm{Kr}$ and the $\mathrm{NaCl}$ lattice provide an instructive selection to use the pair-distribution functions to compare and contrast two solids with the same lattice parameters. The pair-distribution functions provide a graphic illustration of how much more open the neutral $\mathrm{Kr}$ lattice is compared with the ionic lattice of $\mathrm{NaCl}$.

\section{Note}

1. The expression "zero-net-charge" (21) is used rather than "charge neutral" to avoid confusion that may arise when the pairdistribution functions of the van der Waals solids, composed of neutral atoms (such as the rare gases) or neutral molecules, are considered.

\section{Literature Cited}

1. Douglas, B.; McDaniel, D. H.; Alexander, J.J. Concepts and Models of Inorganic Chemistry, 3rd ed.; Wiley: New York, 1994; p 224.

2. McQuarrie, D. A. Statistical Mechanics, 1st ed., Harper and Row: New York, 1976; p 258.

3. Verlet, L. Phys. Rev. 1968, 165, 201-214.

4. Borwein, D.; Borwein, J. M.; Taylor, K. F. J. Math. Phys. 1985, 26, 2999-3009.

5. Kihara, T.; Koba, S. J. Phys. Soc. Japan 1952, 7, 348-354.

6. Buhler, J.; Wagon, S. Math. Educ. Res.1996, 5, 49-55.

7. Crandall, R. E. Exp. Math. 1999, 8, 367-379.

8. Grosso, R. P., Jr.; Fermann, J. T.; Vining, W. J. J. Chem. Educ. 2001, 78, 1198-1202.

9. Burrows, E. L.; Kettle, S. F. A. J. Chem. Educ. 1975, 52, 58-59.

10. Quane, E. L.J. Chem. Educ. 1970, 47, 396-398.

11. Vining, W. J.; Grosso, R. P., Jr.; Fermann, J. T. J. Chem. Educ. 2003, 80, 108.

12. Winn, J. S. Physical Chemistry, 1st ed.; Harper-Collins: New York, 1995; p 604.

13. Kittel, C. Introduction to Solid State Physics, 5th ed.; Wiley: New York, 1976; p 90.

14. Evjen, H. M. Phys. Rev. 1932, 39, 675-687.

15. Handbook of Chemistry and Physics, 73rd ed., Lide, D. R., Ed.; CRC: Boca Raton, FL, 1995; Section 12-31.

16. Woan, G. The Cambridge Handbook of Physics Formulas; Cambridge University Press: Cambridge, 2002; p 9.

17. "Genplot-A Data Analysis/Graphics Program for Scientists and Engineers" from Computer Graphics Solutions. http://www.genplot.com/download.htm (accessed Oct 2009).

18. Shuo, Z.; Nanxian, C. Phys. Rev. B 2002, 66, 064106-1064106-10.

19. Kerins, P. N.; McCaffrey, J. G. J. Chem. Phys. 1998, 109, 3131-3136.

20. Ryan, M. Matrix-Isolation of Atomic Sodium in Rare Gas Solids-A Combined Luminescence Spectroscopy and Molecular Dynamics Study. Ph.D. Thesis; National University of IrelandMaynooth, Ireland, 2007. Ryan, M.; Collier, M.; de Pujo, P.; Crepin, C.; McCaffrey, J. G. J. Phys. Chem A 2009, DOI 10.1021/ jp905596a.

21. Slater, J. C. Insulators Semiconductors and Metals Statistical Mechanics: Quantum Theory of Molecules and Solids; McGraw Hill: New York, 1967; Vol. 3, pp 215-220.

\section{Supporting JCE Online Material}

http://www.jce.divched.org/Journal/Issues/2009/Dec/abs1450.html

Abstract and keywords

Full text (PDF) with links to cited URL and JCE articles

Supplement: Appendices A-C 